OPEN ACCESS

Edited by:

Graça Soveral,

Universidade de Lisboa, Portugal

Reviewed by:

Marçal Pastor-Anglada,

Universitat de Barcelona, Spain

Irene Abreu Rodríguez,

Universität Zürich, Switzerland

Corinna Burger,

University of Wisconsin-Madison,

United States

${ }^{*}$ Correspondence:

Ana Galán-Cobo

agalan@mdanderson.org

Miriam Echevarría

irusta@us.es

Specialty section:

This article was submitted to

Cellular Biochemistry,

a section of the journal

Frontiers in Molecular Biosciences

Received: 22 January 2018 Accepted: 13 April 2018

Published: 03 May 2018

Citation:

Galán-Cobo A, Arellano-Orden E, Sánchez Silva R, López-Campos JL,

Gutiérrez Rivera C, Gómez

Izquierdo L, Suárez-Luna N,

Molina-Molina M, Rodríguez Portal JA

and Echevarría M (2018) The

Expression of AQP1 IS Modified in

Lung of Patients With Idiopathic

Pulmonary Fibrosis: Addressing a Possible New Target.

Front. Mol. Biosci. 5:43.

doi: 10.3389/fmolb.2018.00043

\section{The Expression of AQP1 IS Modified in Lung of Patients With Idiopathic Pulmonary Fibrosis: Addressing a Possible New Target}

\author{
Ana Galán-Cobo ${ }^{1 *}$, Elena Arellano-Orden ${ }^{1}$, Rocío Sánchez Silva ${ }^{1,}$ \\ José Luis López-Campos 2,3, César Gutiérrez Rivera ${ }^{2}$, Lourdes Gómez Izquierdo ${ }^{4}$, \\ Nela Suárez-Luna ${ }^{1}$, María Molina-Molina ${ }^{3,5}$, José A. Rodríguez Portal ${ }^{2}$ and \\ Miriam Echevarría ${ }^{1 *}$
}

'Departamento de Fisiología Médica y Biofísica, Instituto de Biomedicina de Sevilla, Hospital Universitario Virgen del Rocío, CSIC, Universidad de Sevilla, Sevilla, Spain, ${ }^{2}$ Unidad Médico-Quirúrgica de Enfermedades Respiratorias, Hospital Universitario Virgen del Rocio, Sevilla, Spain, ${ }^{3}$ Centro de Investigación Biomédica en Red sobre Enfermedades Respiratorias (CIBERES), Madrid, Spain, ${ }^{4}$ Servicio Anatomía Patológica, HU Virgen del Rocío Sevilla, Seville, Spain, ${ }^{5}$ Laboratorio de Neumologia Experimental, Servicio de Neumologia, Institut d'Investigació Biomédica de Bellvitge, Hospital Universitario de Bellvitge, Barcelona, Spain

Activation of the epithelial-mesenchymal transition process (EMT) by which alveolar cells in human lung tissue undergo differentiation giving rise to a mesenchymal phenotype (fibroblast/miofibroblasts) has been well recognized as a key element in the origin of idiopathic pulmonary fibrosis (IPF). Here we analyzed expression of AQP1 in lung biopsies of patients diagnosed with IPF, and compared it to biopsies derived from patients with diverse lung pneumonies, such as hypersensitivity pneumonitis, sarcoidosis or normal lungs. Immunostaining for AQP1 showed a clear increment of AQP1 localized in the alveolar epithelium in biopsies from IPF patients alone. Moreover, to examine the possible participation of AQP1 in the pathophysiology of IPF, we evaluated its role in the pro-fibrotic transformation induced by transforming growth factor (TGF- $\beta$ ) in vitro. Human alveolar epithelial cells (A549), and fibroblasts derived from an IPF patient (LL29), or fibroblasts from healthy normal lung tissue (MRC-5), were treated with TGF- $\beta$, and levels of expression of AQP1, as well as those of E-cadherin, vimentin, $\alpha$-SMA and collagen were analyzed by RT-qPCR, western blot and immunohistochemistry. An increase of AQP1 mRNA and protein after TGF- $\beta$ treatment (4-72h) was observed either in A549 or IPF fibroblast-LL29 but not in MRC-5 fibroblasts. A gradual reduction of E-cadherin, and increased expression of vimentin, with no changes in $\alpha$-SMA levels were observed in A549. Whereas in LL29 and MRC-5, TGF- $\beta 1$ elicited a large production of collagen and $\alpha$-SMA that was significantly greater in IPF fibroblast-LL29. Changes observed are consistent with activation of EMT by TGF- $\beta$, but whether modifications in AQP1 expression are responsible or independent events occurring at the same time is still unknown. Our results suggest that AQP1 plays a role in the pro-fibrotic TGF- $\beta$ action and contributes to the etiology and pathophysiology of IPF. Understanding AQP1's role will help us comprehend the fate of this disease.

Keywords: interstitial lung disease (ILD), fibrosis, sarcoidosis, aquaporins (AQPs), inflamation, type II pneumocytes, IPF, AQP1 


\section{INTRODUCTION}

Idiopathic pulmonary fibrosis (IPF) is the most widely found interstitial lung disease. IPF is a progressive disease usually fatal, characterized by inability of the alveolar epithelium to repair a lesion. The re-epithelialization fails and transformation to fibroblast/myofibroblast persist, with increase in extracellular matrix deposition and alteration of functional lung architecture, which will finally produce a respiratory failure (Lynch et al., 2006; Raghu et al., 2011). Clinical IPF is normally associated in surgical lung biopsy with a histopathological pattern of usual interstitial pneumonia (UIP). Though the origin of this pathology remains not fully understood, (Willis and Borok, 2007; Spagnolo et al., 2015) it is postulated that following alveolar epithelial injury, a process of repair and scar formation is initiated that implicates important changes and cell activation. However, in some cases, an alteration of the healing process occurs and lung fibrosis develops (Willis et al., 2006). In IPF, activated epithelial cells and recruited inflammatory cells release potent fibrogenic growth factors and cytokines that favor fibroblasts proliferation and its transformation to myofibroblasts (Khalil et al., 2005; Takai et al., 2013). The activation of an epithelial-mesenchymal transition (EMT) process by which alveolar epithelial cells (AEC) undergo a transition or trans-differentiation process to mesenchymal like cells, such as fibroblasts or myofibroblasts, is gaining acceptance as a key element in the origin of this pathology. Both pathological alternative pathways do not exclude each other and may likely coexist in the origin of IPF.

Different fibrogenic growth factors have been clearly implicated as inducers of lung fibrosis, among them the transforming growth factor (TGF- $\beta$ ) is perhaps the most relevant factor associated to lung fibrosis. Furthermore, a crucial role for TGF- $\beta$ has been indicated in the induction of EMT in many physiological scenarios, including for instances in tissue fibrosis (Chapman, 2011; Tirino et al., 2013). It has been demonstrated that TGF- $\beta$ induces in vitro EMT in AEC, and that epithelial and mesenchymal markers co-localize in hyperplastic alveolar type II cells in interstitial pulmonary fibrosis (IPF) tissue, indicating that AEC could show great plasticity and function as a source for new fibro- and myofibroblasts cells in lung fibrosis (Chapman, 2011; Tirino et al., 2013).

Several aquaporins (AQP1, AQP3, AQP4, and AQP5) have been recognized in the respiratory system. Each one presents in a particular location, probably indicating different physiological roles in the pulmonary tissue (Guarino et al., 2009; Camelo et al., 2014). AQP1 is expressed in both membranes, apical and basolateral, of the microvascular endothelium, besides of being expressed in the visceral pleura. AQP3 and AQP4 are expressed in the airway epithelium. AQP5 is expressed in the apical membrane of type-I pneumocytes, and also in the apical side of acinar cells in the submucosal glands of nasopharynx and rest of airways.

In previous studies, we demonstrated that Aquaporin-1 (AQP1) up-regulates its expression in the lung of animals exposed to hypoxia for 24-48 h (Echevarría et al., 2007). We also demonstrated that the transcription factor inducible by hypoxia HIF-1a, partially participates in this process along with other factors in the inflammatory pathway (Abreu-Rodríguez et al.,
2011). We then became interested if the regulation by hypoxia would occur in patients suffering hypoxemic diseases and whether the remodeling of AQP1 expression may play a role in developing pathologies, such as chronic obstructive pulmonary disease (EPOC) (Calero et al., 2014) and IPF (Gutiérrez et al., 2013).

A clear association between expression of AQP1 and increment of cell proliferation in different cellular models has been recently demonstrated (Galán-Cobo et al., 2015, 2016). Moreover, in a murine model of acute lung injury a relationship between ANGII and AQP1 expression was indicated (Cao et al., 2010) and up-regulation of pulmonary AQP1 expression was observed in mice with pulmonary fibrosis induced by bleomycin (Gao et al., 2013), leading authors to propose a possible role for AQP1 in the pathogenesis of lung fibrosis.

Thus, with all these precedents in mind we decided to look for alternative pathways that may explain connections between changes in the AQP1 expression pattern in lung cellular types of patients with IPF and the pathophysiology of the disease. Lung biopsies of patients with different interstitial lung disorders were analyzed by immunohistochemistry for AQP1 expression and experiments in cell lines treated with the pro-fibrotic agent TGF- $\beta 1$ were performed in vitro. The human alveolar epithelial cell line A549, and two different cell lines of human fibroblast, one derived from an IPF patient (LL-29) and the other from healthy fibroblast (MRC5), were treated with TGF- $\beta 1$, and the correlation between expression of AQP1 and other proteins were analyzed to determine their role in the induced EMT or fibroblast-myofibroblast transition process.

\section{MATERIALS AND METHODS}

\section{Human Tissue Samples}

All paraffin-embedded samples were acquired from the Department of Pathology, HUVR of Seville, Spain. Originally, samples were obtained by pulmonary biopsy corresponding to IPF and other fibrotic and non-fibrotic interstitial lung diseases (ILD). For the control group we took samples of patients undergoing surgery for spontaneous pneumothorax otherwise healthy. The number of groups, as well as the number of samples included per group in the analysis are indicated in Table 1, and are as follow: (20) idiopatic pulmonary fibrosis-usual interstitial pneumonia (IPF-UIP), (2) non-specific interstitial pneumonia (NSIP), (1) cryptogenic organizing pneumonia (COP), (5) IPF-not otherwise specified, (6) hypersensitivity pneumonitis, (4) sarcoidosis and (7) control lungs. Written informed consent was obtained from all participants. The study followed the tenets of the WMA Declaration of Helsinki for research involving human subjects and was approved by the ethics committee of the University Hospital Virgen del Rocío (HUVR).

\section{Immunohistochemistry of Biopsies}

All samples examined were obtained from formalinfixed, paraffin-embedded pieces. Slices of $5 \mu \mathrm{m}$ were cut with a microtome and mounted on microscope slides. Immunohistochemical samples were obtained from paraffin 
sections that were immersed in xylene and rehydrated through a series of decreasing dilutions of ethanol. Blocking of endogenous peroxidase, epitope retrieval and immunostaining procedure were done as previously reported (López-Campos et al., 2011). Primary antibody, rabbit polyclonal anti-AQP1, (1:500 dilution, Abcam, Cambridge, UK) was used overnight, for the developing of brown precipitates we then used the two steps system EnVision + Dual Link System-HRP (DakoCytomation, Dako Denmark). Qualitative analysis was made by two independent observers. We assigned zero for no staining on the sample, low positive $(<25 \%$ of the sample stained), medium positive $(50 \%$ of the sample stained) and high positive ( $>75 \%$ of the sample stained) values (Tables 1, 2). Immunoreactivity was analyzed in terms of surface and type of cells showing AQP1 on their surface. Samples were photographed using an AX70-Olympus microscope equipped with an Olympus DP10 camera (Denmark).

\section{Cell Culture}

A549 cells (Human lung alveolar epithelial) and LL29 (Human idiopathic pulmonary fibrosis) were purchased

TABLE 1 | Summary of interstitial lung diseases (pneumonias) included in the study.

\begin{tabular}{lcc}
\hline & N & (\%) \\
\hline $\begin{array}{lcc}\text { Idiopathic Pulmonary Fibrosis (IPF)/Usual } \\
\text { interstitial pneumonia (IPF-UIP) }\end{array}$ & 20 & 44.4 \\
Non-specific interstitial pneumonia (NSIP) & 2 & 4.4 \\
Cryptogenic organizing pneumonia (COP) & 1 & 2.2 \\
Idiopathic interstitial pneumonia not & 5 & 11.1 \\
otherwise specified & & \\
Hypersensitivity pneumonia & 6 & 13.3 \\
Sarcoidosis & 4 & 15.6 \\
Controls & 7 & 100 \\
\hline Total & 45 &
\end{tabular}

from the American Type Culture Collection (ATCC, Rockville, USA); MRC-5 (Human lung fibroblast cell line) cells were obtained from the European Collection of Cell Culture (ECACC, Salisbury, UK). A549 cells were cultured as monolayers in DMEM:F12 (F12K Nut Mix) supplemented with $10 \%$ heat-inactivated fetal bovine serum, $100 \mathrm{U} / \mathrm{mL}$ penicillin-streptomycin (Invitrogen, Carlsbad, CA, USA). MRC-5 cells were cultured as monolayers in MEM supplemented with $10 \%$ heat-inactivated fetal bovine serum, $100 \mathrm{U} / \mathrm{mL}$ penicillin-streptomycin, and $2 \mathrm{mM} \mathrm{L-}$ glutamine (Invitrogen, Carlsbad, CA, USA). LL-29 cells were cultured in Ham's F12K medium supplemented with $15 \%$ heat-inactive fetal bovine serum, $100 \mathrm{U} / \mathrm{mL}$ penicillin-streptomycin, and $2 \mathrm{mM}$ L-glutamine (Invitrogen, Carlsbad, CA, USA). Cells were cultured at $5 \% \mathrm{CO}_{2}$ at $37^{\circ} \mathrm{C}$.

\section{TGF- $\beta 1$ Assays}

A549 $\left(2.4 \times 10^{5}\right)$, MRC-5 $\left(3 \times 10^{5}\right)$, and LL-29 $\left(2.5 \times 10^{5}\right)$ cells were plated on $60 \mathrm{~mm}$ dishes (Nunc, Roskilde, Denmark), when the cells reached $80 \%$ of confluence we added fresh medium $1 \%$ FBS for $24 \mathrm{~h}$ and then we added $10 \mathrm{ng} / \mathrm{ml}$ of TGF- $\beta 1$ (R\&D System, Minneapolis, MN, USA) for $4,16,24,48$, or $72 \mathrm{~h}$. We used untreated cells as control.

Morphological changes in A549, MRC-5 and LL-29 cells were examined by inverted fluorescence microscopy (Zeiss Axiovert 25, Carl Zeiss Co. Oberkochen, Germany) and photographed using a CCD Canon camera.

\section{Indirect Immunocytochemistry Staining of Cultures Cell}

Cells were seeded into 24-well plates at a concentration of $2 \times 10^{4}$ cells/well. After treatment with $10 \mathrm{ng} / \mathrm{ml} \mathrm{TGF-}$ $\beta 1$ for $24 \mathrm{~h}$ the culture medium was discarded and cells were washed with $\mathrm{Ca}^{++}-\mathrm{Mg}^{++}$PBS $1 \mathrm{x}$ and fixed with $3 \%$ paraformaldehyde for $10 \mathrm{~min}$ at room temperature. Cells were

TABLE 2 | Association between AQP1 staining and interstitial pneumonias.

\begin{tabular}{|c|c|c|c|c|c|}
\hline & \multicolumn{4}{|c|}{ Type II pneumocytes AQP1 immunoreactivity } & \multirow[t]{2}{*}{$P$-value* } \\
\hline & Negative & Low positive & Medium positive & High positive & \\
\hline $\begin{array}{l}\text { Usual interstitial } \\
\text { pneumonia }(n=20)\end{array}$ & $2(10 \%)$ & $1(5 \%)$ & $8(40 \%)$ & $9(45 \%)$ & 0.001 \\
\hline $\begin{array}{l}\text { Non-specific interstitial } \\
\text { pneumonia }(n=2)\end{array}$ & - & - & $1(50 \%)$ & $1(50 \%)$ & 0.087 \\
\hline $\begin{array}{l}\text { Cryptogenic organizing } \\
\text { pneumonia }(n=1)\end{array}$ & - & - & $1(100 \%)$ & - & 0.249 \\
\hline $\begin{array}{l}\text { Idiopathic interstitial } \\
\text { pneumonia not otherwise } \\
\text { specified }(n=5)\end{array}$ & $4(80 \%)$ & - & $1(20 \%)$ & - & 0.685 \\
\hline $\begin{array}{l}\text { Hypersensivity } \\
\text { pneumonia }(n=6)\end{array}$ & $3(50 \%)$ & $3(50 \%)$ & - & - & 0.266 \\
\hline Sarcoidosis $(n=4)$ & $4(100 \%)$ & - & - & - & 1 \\
\hline Control $(n=7)$ & $6(85.7 \%)$ & 1 (14.3\%) & - & - & - \\
\hline
\end{tabular}

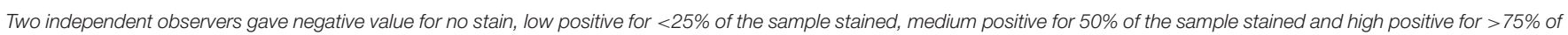

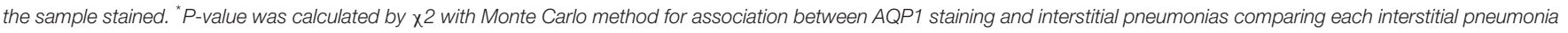
against the control group and using the Bonferroni correction for multiple comparisons $(p<0.008)$ only the group of usual interstitial pneumonia resulted different from control $(p=0.001)$. 
washed and permeabilized/blocked with $0.1 \%$ Triton X-100 in $10 \%$ Fetal Calf Serum (FCS) solution for $10 \mathrm{~min}$ at room temperature and incubated with primary antibodies; 1:200 of E-cadherin (BD Biosciences, San Jose, CA, USA) and 1:100 of AQP1 (Abcam, Cambridge, UK) overnight at $4^{\circ} \mathrm{C}$. After washing with PBS cells were then incubated with Alexa 488 donkey anti-mouse (Invitrogen, Carlsbad, CA, USA) 1:200 in blocking solution for AQP1 and E-cadherin staining for $1 \mathrm{~h}$ at room temperature. F-actin was labeled with red-fluorescent Alexa Fluor 594 Phalloidin (Thermo Fisher Scientific, Waltham, MA, USA) 1:1000 for $10 \mathrm{~min}$ at room temperature. Samples were washed with PBS and then mounted with DAKO (Invitrogen, Carlsbad, CA, USA) and preserved at $4^{\circ} \mathrm{C}$. Immunocytochemestries were photographed using a fluorescence microscope Olympus BX 71 with a refrigerated camera DP70.

\section{Western Blotting}

Plated cells were washed with cold PBS, collected by scraping in $1 \mathrm{ml}$ of cold PBS and centrifuged at $300 \mathrm{~g}$ for $5 \mathrm{~min}$ at $4^{\circ} \mathrm{C}$. For whole-cell protein extract, the pellet was lysed in a variable volume of homogenization buffer containing: $50 \mathrm{mM}$ Hepes (pH 7.3); 5 mM EDTA; 250 mM NaCl; 5 mM DTT; $0.2 \%$ (v/v) NP40 (Sigma-Aldrich); and 1\% (v/v) Complete Protease Inhibitors Cocktail (Sigma-Aldrich). The resuspended pellet was left on ice for 5-15 min, vortexed, and then centrifuged at $16,000 \mathrm{~g}$ for $15 \mathrm{~min}$ at $4^{\circ} \mathrm{C}$, and proteins contained in the supernatant were stored. Protein concentration was analyzed using the Bradford method (BioRad Protein Assay, BioRad, Berkeley, CA) and kept at $-20^{\circ} \mathrm{C}$ until Western blot analysis (Galán-Cobo et al., 2016). For all proteins analyzed, $30 \mu \mathrm{g}$ of whole-cell extracts were resolved by SDSPAGE on $10-12 \%$ gels. After electrophoresis, proteins were transferred onto PVDF membranes (Hybond-P, Amersham Biosciences, Pittsburgh, PA) using a Novex apparatus (Novel Experimental Technology, San Diego, CA). Membranes were probed with 1:700 anti-AQP1 (Abcam, Cambridge, UK), 1:2500 anti-E-Cadherin (BD Biosciences, San Jose, CA, USA), 1:1500 anti-Vimentin (Abcam, Cambridge, UK), $1: 1000$ anti- $\alpha$-SMA (Abcam, Cambridge, UK) and 1:10000 anti-cyclophilin A (Abcam, Cambridge, UK) antibodies. Immunoreactive bands were developed with the ECL Prime system (Amersham Biosciences) and visualized using a digital imaging system (ImageQuant LAS 4000 Mini, GE Healthcare, Buckinghamshire, UK).

\section{RNA Isolation and Quantitative PCR}

RNA was extracted from cells using TRIzol reagent (Invitrogen, Carlsbad, CA, USA) according with manufacturer protocol. After mRNA isolation, reverse transcription (RT) was performed for $5 \mu \mathrm{g}$ of RNA using SuperScript II RNase-H reverse transcriptase (Invitrogen, Carlsbad, CA, USA). Real-time quantitative PCR (qPCR) analysis was carried out in an ABI Prism 7500 Sequence Detection System using SYBR Green PCR Master Mix (Applied Biosystems, Warrington, UK) and the thermocycler conditions recommended by the manufacturer. The expression of HPRT1 was used to normalize for differences in amounts of input DNA between samples. Primers were designed using the Primer Express software (version 2.0, Applied Biosystems, Warrington, UK) and their sequences are indicated in Table 3. Melting curve analysis showed a single sharp peak with the expected melting temperature for all samples.

\section{Statistical Analysis}

Data analysis was performed using SPSS Advanced Statistics (SPSS Inc., Chicago, Illinois), version 19.0. For the statistical analysis of data derived from analysis by immunohistochemistry of patient biopsies, the " $p$ " value for comparative analysis of association between AQP1 staining and interstitial pneumonias was calculated by $\chi^{2}$ with Monte Carlo method. The Bonferroni correction was used for multiple comparisons and the Fisher exact test when comparing each interstitial pneumonia against the control group. For all experiments performed in cells in culture the data are expressed as mean \pm standard error of the mean from at least four different experiments. The statistical significance for normal distribution data was estimated using one-way ANOVA followed by Bonferroni's post-hoc test to compare more than two groups. Data with non-normal distribution were analyzed using Mann-Whitney $\mathrm{U}$ or Kruskal-Wallis H-test for two or more than two groups, respectively. Values of $p \leq 0.05$ were considered significant.

TABLE 3 | Primer sequences used for the amplification of cDNAs by qPCR.

\begin{tabular}{lcc}
\hline Genes & Forward sequence & Reverse sequence \\
\hline Aquoporin 1 (AQP1) & GGACACCTCCTGGCTATTGACTAC & GTTGCTGAAGTTGTGTGTGATCAC \\
E-Cadherin (CDH1) & TCGACACCCGATTCAAAGTG & GTCCCAGGCGTAGACCAAGA \\
Vimentin $(V I M)$ & TGCCCTTAAAGGACCAATGAG & AGGCGGCCAATAGTGTCTTG \\
$\alpha$-SMA (ACTA2) & CTGTCCAGCCATCCTTCAT & CCGTGATCTCCTTCTGCATT \\
Collagen Type I Alpha 1 (COL1A1) & TGACCGAGACGTGTGGAAC & CAGATCACGTCATCGCACAAC \\
Hypoxanthine & ACTGAACGTCTTGCTCGAGATG & AGCAGGTCAGCAAAGATTTATAGC \\
Phosphoribosyltransferase 1 (HPRT1)
\end{tabular}

Primers were designed using the Primer Express software (Applied-Biosystems). qPCR: quantitative PCR. 


\section{RESULTS}

\section{AQP1 Is Specifically Expressed in Hyperplasic Cuboidal Type II Pneumocytes in the Alveolar Epithelium in Patients With Idiopathic Pulmonary Fibrosis}

In the present study we analyzed expression of AQP1 in lung biopsies of patients diagnosed with IPF, and compare it to biopsies derived from diverse interstitial lung diseases such as others pneumonias, hypersensitivity pneumonitis, sarcoidosis, or normal lungs (Table 1). Conditions for AQP1 antibody immunohistochemistry in human lung biopsies were determined in a previous study (López-Campos et al., 2011). A summary of results from the analysis of biopsies is presented in Table 2. Normal lung tissues is consistent with previous reports (LópezCampos et al., 2011; Calero et al., 2014) in that AQP1 immunostaining was detected predominantly in endothelial cells of alveoli capillaries and the rest of blood vessels; faint labeling was also detected on neumocytes of the alveolar wall (Figure 1). No labeling was detected in absence of the primary antibody (data not shown).

Compared to the rest of pathologies analyzed, the immunohistochemistry assays revealed that only biopsies from IPF show a clear increment of AQP1 staining that localizes remarkably over hyperplasic cuboidal type II pneumocytes in the alveolar epithelium, and to a much lesser grade over few planar, hyperplasic or metaplasic type I cells (Figure 1). A more detailed observation over the fibrosis focus of IPF patients, where the transition from fibroblasts to miofibroblasts occurred, showed that type I epithelial cells lining the fibrotic focus are totally deprived of AQP1 expression. In the rest of the samples basal AQP1 expression was limited to erythrocytes and endothelial cells. Absence of AQP1 labeling was observed over the interstitial lung or in myoepithelial cells. Changes in the expression pattern of $\beta$-catenin and E-cadherin were also studied but need further analysis of the immunoassays. In conclusion, the analysis demonstrates a possible role for $\mathrm{AQP1}$ expression in the reactive process occurring over fibrotic focus of the alveoli epithelium of IPF patients.

\section{AQP1 Is Overexpressed Along Epithelial Mesenchymal Transition (EMT) Induced by TGF- $\beta$}

To further investigate the implication of AQP1 in the initiation and progression of IPF we examined epithelial cells as central to the pathogenesis of IPF. Experiments performed in the human lung epithelial cell line A549 has shown that treatment with the pro-fibrotic agent TGF- $\beta 1(10 \mathrm{ng} / \mathrm{ml})$ produces a time course (24, 48,72 , and $96 \mathrm{~h}$ ) differentiation of the epithelial cells toward a fibroblastic-like phenotype, together with an increase of mRNA levels of the mesenchymal markers vimentin (5-fold) and $\alpha$ SMA (2.5-fold). Consequently, a decrease in the levels of the epithelial marker E-cadherin was found after TGF- $\beta 1$ treatment. Interestingly, levels of AQP1-mRNA were also measured and a

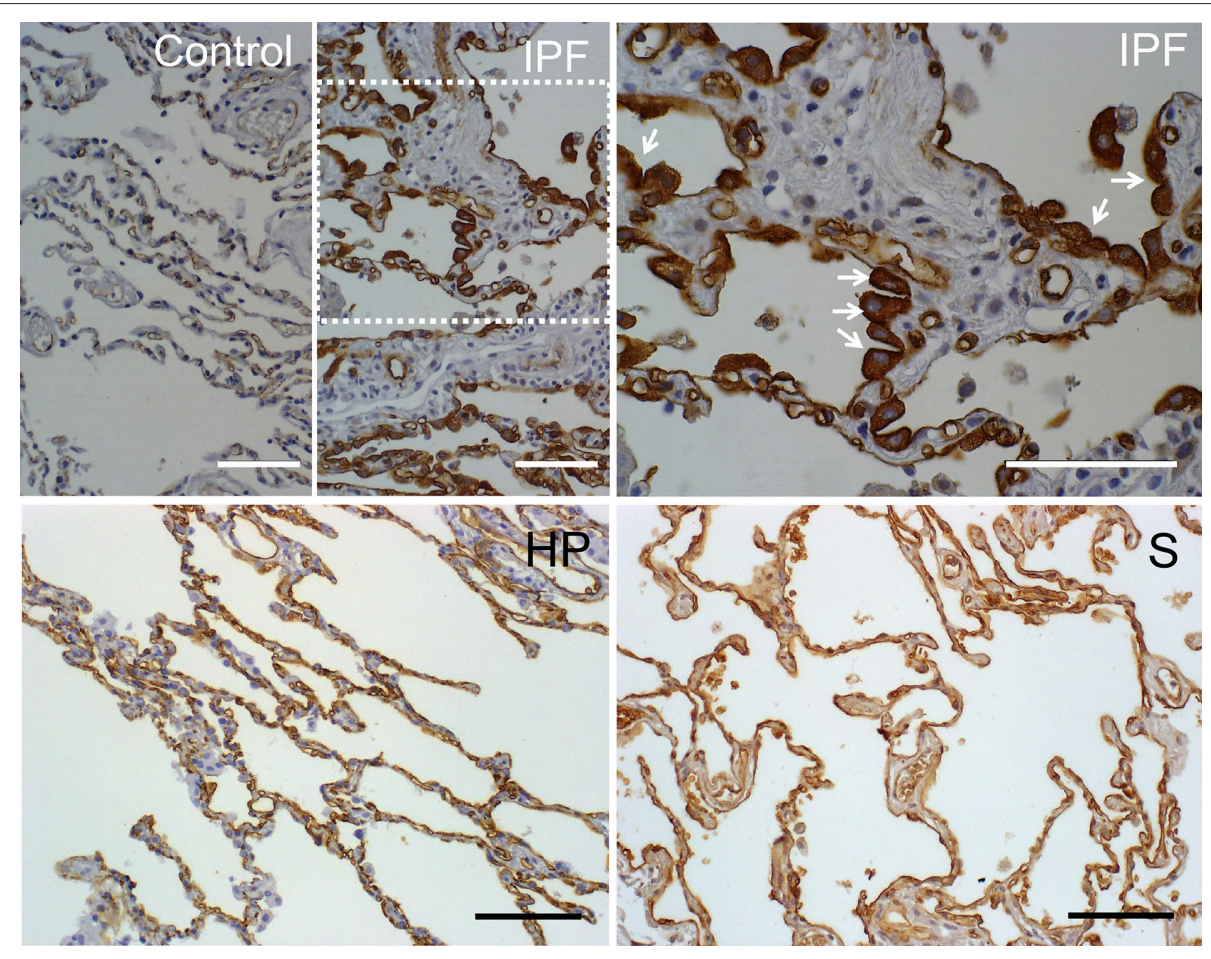

FIGURE 1 | Immunostaining of AQP1 in lung biopsies of patients. Pulmonary parenchyma of a healthy patient is shown as control. In the pulmonary parenchyma of patients with usual intersticial pneumonia (IPF) reactive type II pneumocytes of alveolus showed intense expression of AQP1 (see the arrows in the IPF at larger magnification). In biopsies of patients with hypersensitivity pneumonia (HP) or sarcoidosis (S), the staining of AQP1 was mainly located in capillaries endothelia. Scale bar represents $100 \mu \mathrm{m}$ in each case. Note the different size of bar in each case. 


\section{A}

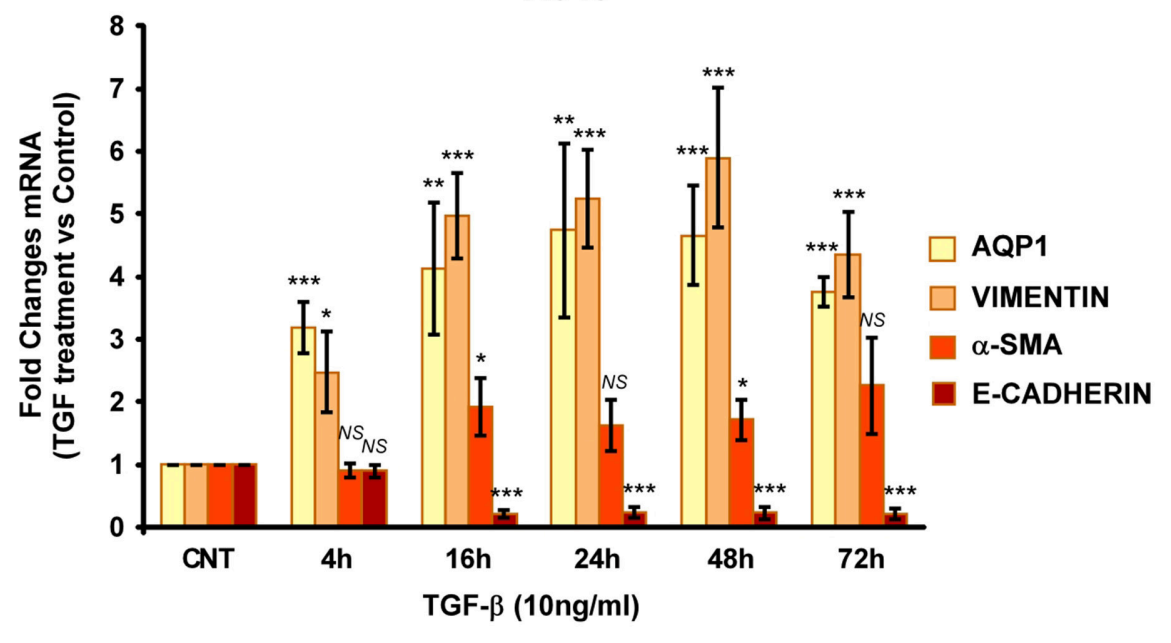

B

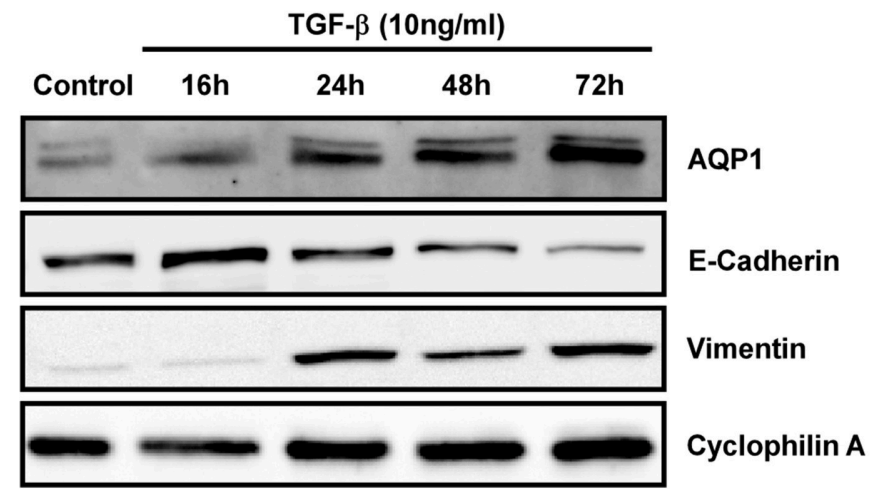

FIGURE 2 | Analysis of EMT induction in A549 cell lines. Cells were treated with TGF- $\beta 1$ (10 ng/ml) for 4, 16, 24, 48, and 72 hours and the mRNA levels of AQP1, E-cadherin, Vimentin and $\alpha$-SMA where quantified by real time RT-PCR analysis (A) and the protein levels by Western blot (B).

substantial increase of AQP1 expression (5-fold) was observed in parallel to the changes described before for vimentin and $\alpha$-SMA (Figure 2A). Similar results to those described for the mRNA, were found for the protein levels of E-cadherin, vimentin and AQP1 (Figure 2B). Immunostaining for AQP1 again showed higher expression levels of AQP1 in the lung epithelial cells A549 after TGF- $\beta 1$ treatment (Figure 3), supporting previous data and indicating a possible role of AQP1 in the EMT process in lung tissue.

We then repeated similar experiments with TGF- $\beta 1$ using two different fibroblast cell lines. A cell line of fibroblasts derived from patients with IPF (LL29) and a cell line of healthy fibroblasts (MRC-5). An increment of the capacity for extracellular matrix production was found in LL29 cells after TGF- $\beta 1$ treatment indicated by higher levels of type I collagen (10-fold) and of $\alpha$ SMA (5-fold). In parallel a strong and stable induction of AQP1 mRNA and protein levels were observed in this cell line obtained from an IPF patient (Figure 4) unlike, in the healthy fibroblast cell line MRC-5. Although similar induction of $\alpha$-SMA and type I collagen were found, AQP1 levels remained unchanged after TGF- $\beta 1$ treatment (Figure 5).
Altogether, these results support the hypothesis that increments of AQP1 expression induced by TGF- $\beta 1$ occur in the lung tissue of patients with IPF. As a result of rising AQP1 in the alveolar epithelia, the cells undergo EMT or fibroblastmyofibroblast transition, thereby contributing to formation of fibroblastic foci and subsequent fibroblast and extracellular matrix accumulation.

\section{DISCUSSION}

Immunohistochemical studies in rats and mice have shown that AQP1 is present in the capillaries and in the sub-epithelial connective tissue of the animal's respiratory tract. Alveolar cells do not express AQP1 on its surface and expression of AQP1 is restricted to the capillary endothelium (Nielsen et al., 1997). At present, the idea remains that AQP1 provides the principal pathway for osmotic transport of water between airspace and lung capillary compartments. Nevertheless, neither alveolar fluid clearance in neonatal and adult lung nor lung fluid accumulation in experimental models of lung injury have reported being affected by AQP1 deletion. 


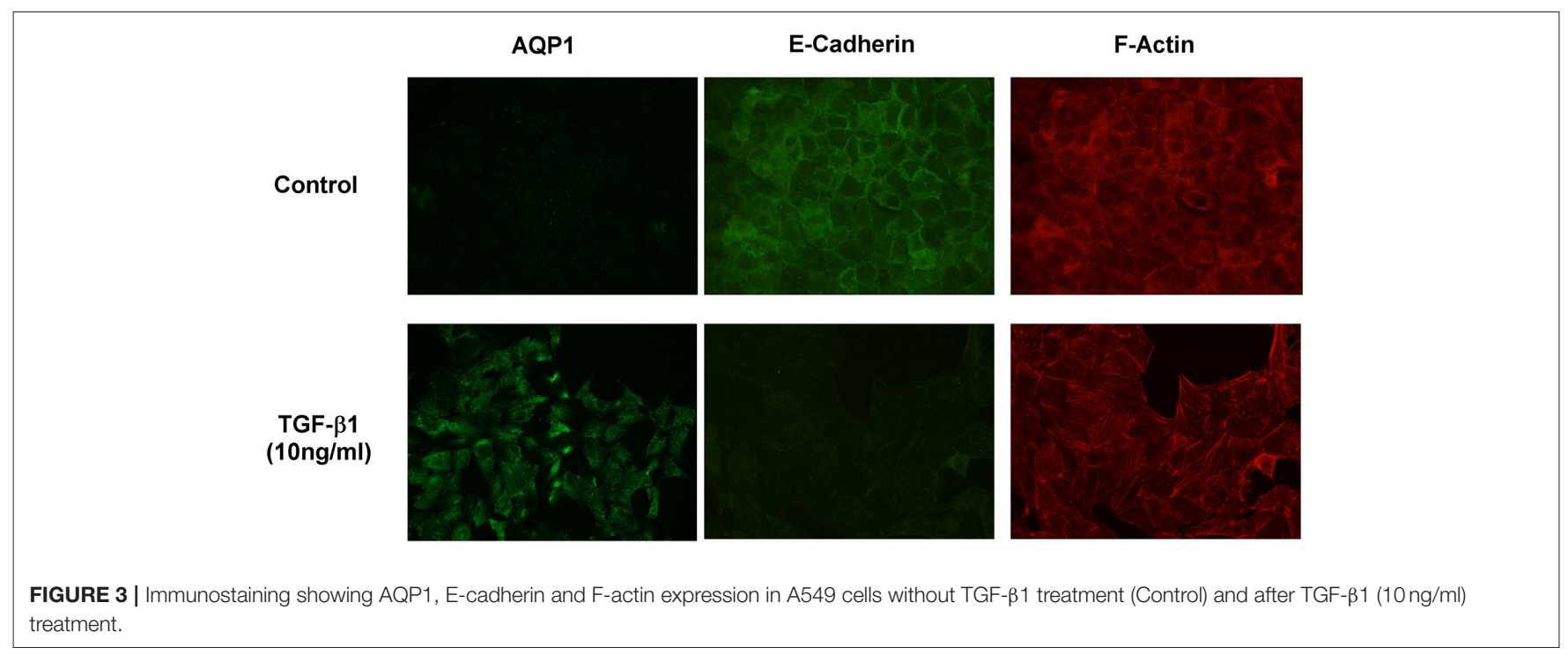

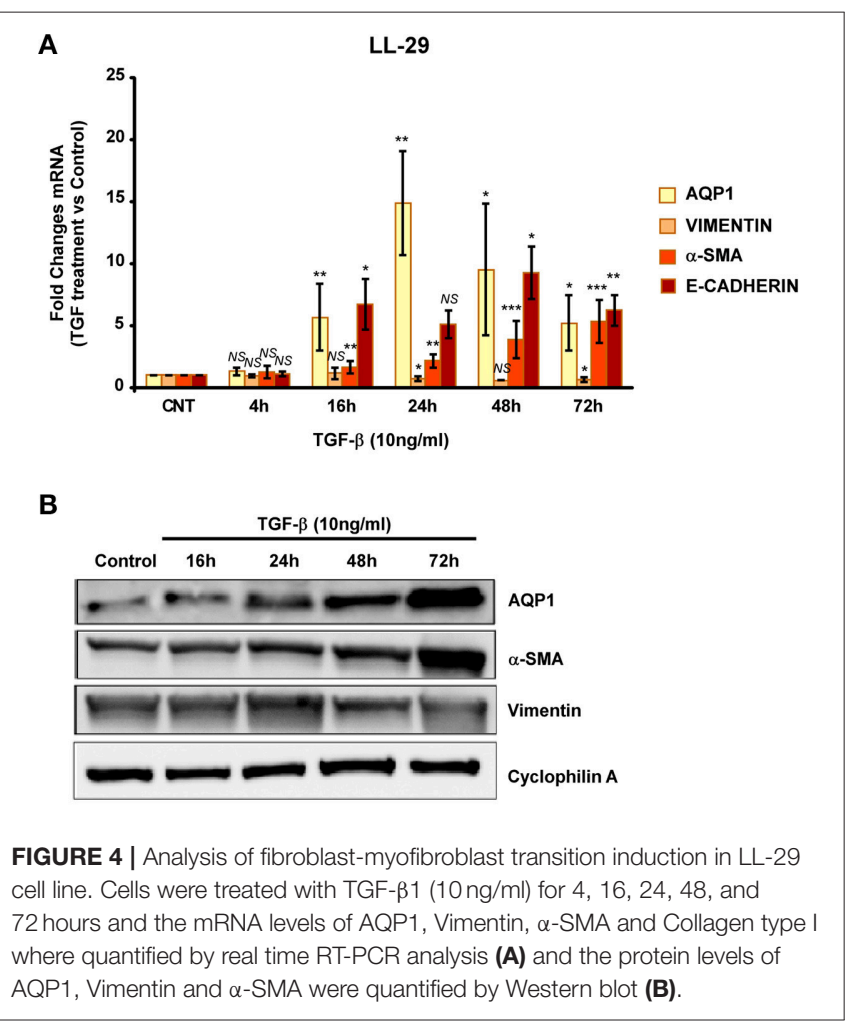

In the present work, we have shown for the first time a large expression of AQP1 in human lung tissue from IPF patients, not only in endothelial cells but also in the alveolar epithelial surface. Alveolar surface is covered by two types of epithelial cells, alveolar cells called type I (AT I) and type II (AT II), also called pneumocytes type I and type II. AT I cells represent $90 \%$ of the alveolar border and are responsible for making gas exchange. AT II cells are characterized by synthesizing and secreting pulmonary surfactant which actives alveolar surface and prevent

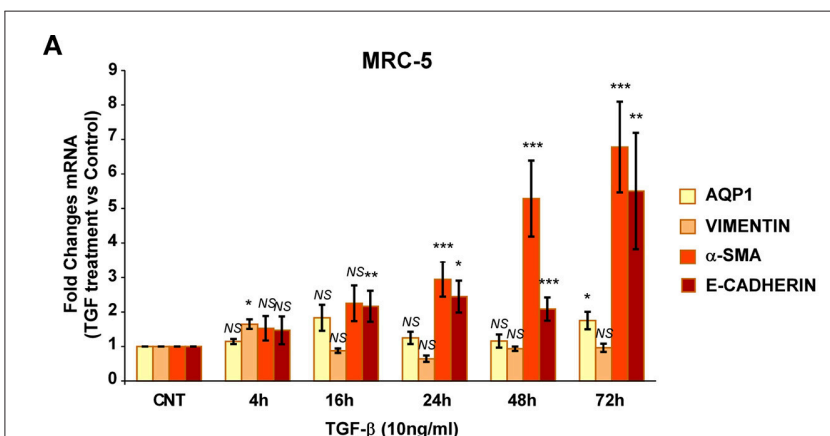

B

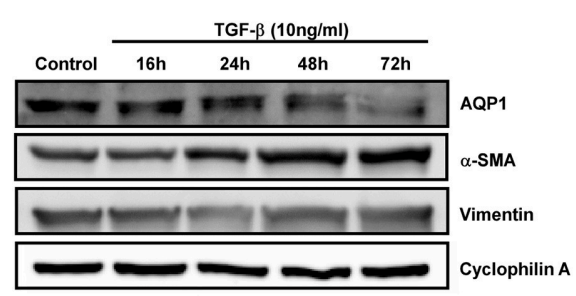

FIGURE 5 | Analysis of fibroblast-myofibroblast transition induction in MRC-5 cell line. Cells were treated with TGF- $\beta 1(10 \mathrm{ng} / \mathrm{ml})$ for $4,16,24,48$, and 72 hours and the mRNA levels of AQP1, Vimentin, $\alpha$-SMA and Collagen type I where quantified by real time RT-PCR analysis (A) and the protein levels of AQP1, Vimentin and $\alpha$-SMA were quantified by Western blot (B).

its collapse among other important functions such as immune defense (Chroneos et al., 2010). AT II cells are progenitors of AT I cells and therefore are responsible for repairing the alveolar epithelium after damage. Under normal conditions, the alveolar epithelial repair occurs through the proliferation of AT II cells and their differentiation into AT I cells. However, IPF is characterized by the fact that both alveolar type II cells and the type I undergo apoptosis and are replaced by fibroblasts (Barbas-Filho et al., 2001) contributing to the disappearance and transformation of the alveolar epithelium. As previously 
described, ATI cells do not express AQP1 (Nielsen et al., 1997). Interestingly, our data shows that expression of AQP1 in the alveolar epithelium was exclusively found in ATII cells.

Analysis performed in different diseases other than IPF show that AQP1 expression is not uniform. This water channel is not expressed in normal lung alveolar cells and is expressed to a much lesser extent in AT II cells from samples obtained from various interstitial lung diseases other than IPF. Even in cases of intense inflammatory activity like hypersensitivity pneumonitis or sarcoidosis, there is not expression of AQP1 in alveolar epithelial cells. The highest expression of AQP1 in type II alveolar epithelial cells occurs significantly in IPF samples. IPF is a progressive disease of unknown etiology (Crystal et al., 2002) that classically presents a histopathological pattern of UIP. The primary characteristics are areas of conventional fibrosis with variable regions of fibroblastic foci, scattered with zones of normal or almost normal lung. Additionally, IPF is also typified by ATII cell hyperplasia (Selman and Pardo, 2003). Although initial studies gave special attention and prevalent role to inflammation on fibrogenesis, the present hypothesis proposes a principal role of the epithelium in the pathogenesis and progression of this disease. Thus, IPF is likely the result of an initial epithelial injury to which the natural repair process fail to repair setting an initial focus of cell activation and damage (Chilosi et al., 2003). Identification of cells in which markers of epithelial and mesenchymal phenotypes are co-expressed, seen both in human lung and in cultures in vitro, sustain the paradigm by which alveolar epithelial cells may serve as a supply of myofibroblasts in lung fibrosis (Willis et al., 2005). Currently, activation and apoptosis of epithelial cells is considered one of the initial events in the development of IPF (Sisson et al., 2010).

In IPF, proliferation and migration are two important events in the alveolar epithelium transformation. Interestingly, AQP1 has been implicated in cell proliferation and migration promotion in lung among other proliferative tissue models (Saadoun et al., 2005; Wei and Dong, 2015; Wu et al., 2015; Galán-Cobo et al., 2016; Tomita et al., 2017; Wang et al., 2017; Yun et al., 2017). On the other hand, EMT is a key event in the IPF progression, which promotes accumulation of fibroblasts and deposition of extracellular matrix in response to epithelial injury. TGF- $\beta$ signaling is considered a key activator of EMT and fibroblast activation in this disease (Chapman, 2011; Tirino et al., 2013). Migration is an essential process during EMT, and although AQP1 has not been directly related with EMT, it is well know that this protein helps the cell migration process by facilitating water influx at the tip of the lamellipodium, resulting in a membrane protusion (Saadoun et al., 2005), and by interacting with $\beta$-catenin (Monzani et al., 2009; Yun et al., 2017). Other AQPs, such as AQP3 and AQP5, activate this process as well (Chen et al., 2014, 2017). Therefore, the large increase in expression of AQP1 we observed on AT II cells in IPF patient

\section{REFERENCES}

Abreu-Rodríguez, I., Sánchez Silva, R., Martins, A. P., Soveral, G., Toledo-Aral, J. J., López-Barneo, J., et al. (2011). Functional and transcriptional induction of biopsies may be crucial in supporting the high proliferation and migration events during the alveolar epithelium transformation. The strong induction by TGF- $\beta 1$ of AQP1 expression specially observed in AECs (A549) in transition to a myofibroblast-like phenotype cell, or in the fibroblasts derived from an IPF patient (LL29), might be among the initial actions triggered by this potent fibrotic agent.

The initial injury which causes lesions in epithelial cells that lead to pulmonary fibrosis are unknown. For the very first time, we have demonstrated increased expression of AQP1 in type II alveolar epithelial cells in IPF patient samples. The increased expression of AQP1, its possible relationship with hyperplasia, and the subsequent insult of ATII remains unclear. Although deeper analysis are required to elucidate the role of AQP1 in the origin of IPF, our findings suggest AQP1 plays an important role in disease progression of IPF patients and should be further explored.

\section{CONCLUSIONS}

The alveolar epithelium of a normal human lung does not express AQP1. However, our studies indicate that IPF patient's hyperplasic type II pneumocytes show an increase in AQP1 expression on their surface. We investigated the role of TGF$\beta 1$, in the epithelial to mesenchymal transition of alveolar epithelial cells and lung fibroblast in IPF patient samples and also confirmed the induction of AQP1 expression. Therefore, the appearance of hyperplastic cells and regulation of AQP1 are implicated in the physiopathology of IPF.

\section{AUTHOR CONTRIBUTIONS}

AG-C and EA-O: Made all molecular biology experiments with culture cells; RS-S and NS-L: Carried out the immunoassays over patient's biopsies; JL-C, CG, MM-M, and JR: Selected, treated and diagnosed the patients included in the analysis; LG: Revised and interpreted immune results in patient's biopsies; ME and AG-C: Conceived and designed the experiments and wrote the manuscript; ME and JR: Contributed reagents, materials, and analysis tools. All authors read and approved the final manuscript.

\section{ACKNOWLEDGMENTS}

We are thankful to all patients which samples were included in this work. This study has been supported by the grants PI12/01882 and PI16/00493 from the Spanish Ministry of Economy and Competitiveness, co-funded by ISCIII and FEDER funds, conceded to ME, and grants from Neumosur and SEPAR foundations given to JR. We thank Juan Manuel Praena for his help with statistical analysis of data. aquaporin-1 gene by hypoxia; analysis of promoter and role of Hif-1 $\alpha$. PLoS ONE 6:e28385. doi: 10.1371/journal.pone.0028385

Barbas-Filho, J. V., Ferreira, M. A., Sesso, A., Kairalla, R. A., Carvalho, C. R., and Capelozzi, V. L. (2001). Evidence of type II pneumocyte apoptosis 
in the pathogenesis of idiopathic pulmonary fibrosis (IFP)/usual interstitial pneumonia (UIP). J. Clin. Pathol. 54, 132-138. doi: 10.1136/jcp.54.2.132

Calero, C., López-Campos, J. L., Izquierdo, L. G., Sánchez-Silva, R., LópezVillalobos, J. L., Sáenz-Coronilla, F. J., et al. (2014). Expression of aquaporins in bronchial tissue and lung parenchyma of patients with chronic obstructive pulmonary disease. Multidiscip. Respir. Med. 9:29. doi: 10.1186/2049-6958-9-29

Camelo, A., Dunmore, R., Sleeman, M. A., and Clarke, D. (2014). The epithelium in idiopathic pulmonary fibrosis: breaking the barrier. Front. Pharmacol. 4:173. doi: $10.3389 /$ fphar.2013.00173

Cao, C. S., Yin, Q., Huang, L., Zhan, Z., Yang, J. B., and Xiong, H. W. (2010). Effect of angiotensin II on the expression of aquaporin 1 in lung of rats following acute lung injury. Zhongguo Wei Zhong Bing Ji Jiu Yi Xue 22, 426-429.

Chapman, H. A. (2011). Epithelial-mesenchymal interactions in pulmonary fibrosis. Annu. Rev. Physiol. 73, 413-435. doi: 10.1146/annurev-physiol-012110-142225

Chen, C., Ma, T., Zhang, C., Zhang, H., Bai, L., Kong, L., et al. (2017). Downregulation of aquaporin 5-mediated epithelial-mesenchymal transition and anti-metastatic effect by natural product Cairicoside $\mathrm{E}$ in colorectal cancer. Mol. Carcinog. 56, 2692-2705. doi: 10.1002/mc.22712

Chen, J., Wang, T., Zhou, Y. C., Gao, F., Zhang, Z.-H., Xu, H., et al. (2014). Aquaporin 3 promotes epithelial-mesenchymal transition in gastric cancer. $J$. Exp. Clin. Cancer Res. 33:38. doi: 10.1186/1756-9966-33-38

Chilosi, M., Poletti, V., Zamò, A., Lestani, M., Montagna, L., Piccoli, P., et al. (2003). Aberrant Wnt/ $\beta$-catenin pathway activation in idiopathic pulmonary fibrosis. Am. J. Pathol. 162, 1495-1502. doi: 10.1016/S0002-9440(10)64282-4

Chroneos, Z. C., Sever-Chroneos, Z., and Shepherd, V. L. (2010). Pulmonary surfactant: an immunological perspective. Cell. Physiol. Biochem. 25, 13-26. doi: $10.1159 / 000272047$

Crystal, R. G., Bitterman, P. B., Mossman, B., Schwarz, M. I., Sheppard, D., Almasy, L., et al. (2002). Future research directions in idiopathic pulmonary fibrosis. Am. J. Respir. Crit. Care Med. 166, 236-246. doi: 10.1164/rccm.2201069

Echevarría, M., Muñoz-Cabello, A. M., Sánchez-Silva, R., Toledo-Aral, J. J., and López-Barneo, J. (2007). Development of cytosolic hypoxia and hypoxiainducible factor stabilization are facilitated by aquaporin-1 expression. J. Biol. Chem. 282, 30207-30215. doi: 10.1074/jbc.M702639200

Galán-Cobo, A., Ramírez-Lorca, R., Serna, A., and Echevarría, M. (2015). Overexpression of AQP3 modifies the cell cycle and the proliferation rate of mammalian cells in culture. PLoS ONE 10:e0137692. doi: 10.1371/journal.pone. 0137692

Galán-Cobo, A., Ramírez-Lorca, R., Toledo-Aral, J. J., and Echevarría, M. (2016). Aquaporin-1 plays important role in proliferation by affecting cell cycle progression. J. Cell. Physiol. 231, 243-256. doi: 10.1002/jcp.25078

Gao, X., Wang, G., Zhang, W., Peng, Q., Xue, M., and Jinhong, H. (2013). Expression of pulmonary aquaporin 1 is dramatically upregulated in mice with pulmonary fibrosis induced by bleomycin. Arch. Med. Sci. 9, 916-921. doi: 10.5114/aoms.2012.31011

Guarino, M., Tosoni, A., and Nebuloni, M. (2009). Direct contribution of epithelium to organ fibrosis: epithelial-mesenchymal transition. Hum. Pathol. 40, 1365-1376. doi: 10.1016/j.humpath.2009.02.020

Gutiérrez, C., Donate, Á., Gómez Izquierdo, L., Molina-Molina, M., Echevarría, M., and Rodriguez Portal, J. A. (2013). Aquoaporin-1 expression in idiopathic pulmonary fibrosis. Eur. Respir. J. 42(Suppl. 57):P2337.

Khalil, N., Xu, Y. D., O'Connor, R., and Duronio, V. (2005). Proliferation of pulmonary interstitial fibroblasts is mediated by transforming growth factor- $\beta 1$-induced release of extracellular fibroblast growth factor- 2 and phosphorylation of p38 MAPK and JNK. J. Biol. Chem. 280, 43000-43009. doi: 10.1074/jbc.M510441200

López-Campos, J. L., Sánchez Silva, R., Gómez Izquierdo, L., Márquez, E., Ortega Ruiz, F., Cejudo, P., et al. (2011). Overexpression of aquaporin-1 in lung adenocarcinomas and pleural mesotheliomas. Histol. Histopathol. 26, 451-459. doi: 10.14670/HH-26.451

Lynch, J. P., Saggar, R., Weigt, S. S., Zisman, D. A., and White, E. S. (2006). Usual interstitial pneumonia. Semin. Respir. Crit. Care Med. 27, 634-651. doi: 10.1055/s-2006-957335

Monzani, E., Bazzotti, R., Perego, C., and La Porta, C. A. M. (2009). AQP1 is not only a water channel: it contributes to cell migration through Lin7/Betacatenin. PLoS ONE 4:e6167. doi: 10.1371/journal.pone.0006167

Nielsen, S., King, L. S., Christensen, B. M., and Agre, P. (1997). Aquaporins in complex tissues. II. Subcellular distribution in respiratory and glandular tissues of rat. Am. J. Physiol. 273(5 Pt 1), C1549-61.

Raghu, G., Collard, H. R., Egan, J. J., Martinez, F. J., Behr, J., Brown, K. K., et al. (2011). An official ATS/ERS/JRS/ALAT statement: idiopathic pulmonary fibrosis: evidence-based guidelines for diagnosis and management. Am. J. Respir. Crit. Care Med. 183, 788-824. doi: 10.1164/rccm.2009-040GL

Saadoun, S., Papadopoulos, M. C., Hara-Chikuma, M., and Verkman, A. S. (2005). Impairment of angiogenesis and cell migration by targeted aquaporin-1 gene disruption. Nature 434, 786. doi: 10.1038/nature 03460

Selman, M., and Pardo, A. (2003). The epithelial/fibroblastic pathway in the pathogenesis of idiopathic pulmonary fibrosis. Am. J. Respir. Cell Mol. Biol. 29, S93-S97.

Sisson, T. H., Mendez, M., Choi, K., Subbotina, N., Courey, A., Cunningham, A., et al. (2010). Targeted injury of type II alveolar epithelial cells induces pulmonary fibrosis. Am. J. Respir. Crit. Care Med. 181, 254-263. doi: $10.1164 / \mathrm{rccm} .200810-1615 \mathrm{OC}$

Spagnolo, P., Sverzellati, N., Rossi, G., Cavazza, A., Tzouvelekis, A., Crestani, B., et al. (2015). Idiopathic pulmonary fibrosis: an update. Ann. Med. 47, 15-27. doi: $10.3109 / 07853890.2014 .982165$

Takai, E., Tsukimoto, M., and Kojima, S. (2013). TGF- $\beta 1$ Downregulates COX-2 expression leading to decrease of PGE2 production in human lung cancer A549 cells, which is involved in fibrotic response to TGF- $\beta 1$. PLOS ONE 8:e76346. doi: 10.1371/journal.pone.0076346

Tirino, V., Camerlingo, R., Bifulco, K., Irollo, E., Montella, R., Paino, F., et al. (2013). TGF- $\beta 1$ exposure induces epithelial to mesenchymal transition both in CSCs and non-CSCs of the A549 cell line, leading to an increase of migration ability in the CD133+ A549 cell fraction. Cell Death Dis. 4:e620. doi: 10.1038/cddis.2013.144

Tomita, Y., Dorward, H., Yool, J. A., Smith, E., Townsend, R. A., Price, J. T., et al. (2017). Role of aquaporin 1 signalling in cancer development and progression. Int. J. Mol. Sci. 18:299. doi: 10.3390/ijms18020299

Wang, Y., Fan, Y., Zheng, C., and Zhang, X. (2017). Knockdown of AQP1 inhibits growth and invasion of human ovarian cancer cells. Mol. Med. Rep. 16, 5499-5504. doi: 10.3892/mmr.2017.7282

Wei, X., and Dong, J. (2015). Aquaporin 1 promotes the proliferation and migration of lung cancer cell in vitro. Oncol. Rep. 34, 1440-1448. doi: 10.3892/or.2015.4107

Willis, B. C., and Borok, Z. (2007). TGF- $\beta$-induced EMT: mechanisms and implications for fibrotic lung disease. Am. J. Physiol. Lung Cell. Mol. Physiol. 293, L525-L534. doi: 10.1152/ajplung.00163.2007

Willis, B. C., duBois, R. M., and Borok, Z. (2006). Epithelial origin of myofibroblasts during fibrosis in the lung. Proc. Am. Thorac. Soc. 3, 377-382. doi: 10.1513/pats.200601-004TK

Willis, B. C., Liebler, J. M., Luby-Phelps, K., Nicholson, A. G., Crandall, E. D., du Bois, R. M., et al. (2005). Induction of epithelial-mesenchymal transition in alveolar epithelial cells by transforming growth factor- $\beta 1$ : potential role in idiopathic pulmonary fibrosis. Am. J. Pathol. 166, 1321-1332. doi: 10.1016/S0002-9440(10)62351-6

Wu, Z., Li, S., Liu, J., Shi, Y., Wang, J., Chen, D., et al. (2015). RNAimediated silencing of AQP1 expression inhibited the proliferation, invasion and tumorigenesis of osteosarcoma cells. Cancer Biol. Ther. 16, 1332-1340. doi: 10.1080/15384047.2015.1070983

Yun, X., Jiang, H., Lai, N., Wang, J., and Shimoda, L. A. (2017). Aquaporin 1mediated changes in pulmonary arterial smooth muscle cell migration and proliferation involve $\beta$-catenin. Am. J. Physiol. Lung Cell. Mol. Physiol. 313, L889-L898. doi: 10.1152/ajplung.00247.2016

Conflict of Interest Statement: The authors declare that the research was conducted in the absence of any commercial or financial relationships that could be construed as a potential conflict of interest.

Copyright (c) 2018 Galán-Cobo, Arellano-Orden, Sánchez Silva, López-Campos, Gutiérrez Rivera, Gómez Izquierdo, Suárez-Luna, Molina-Molina, Rodríguez Portal and Echevarría. This is an open-access article distributed under the terms of the Creative Commons Attribution License (CC BY). The use, distribution or reproduction in other forums is permitted, provided the original author(s) and the copyright owner are credited and that the original publication in this journal is cited, in accordance with accepted academic practice. No use, distribution or reproduction is permitted which does not comply with these terms. 